Research Article

\title{
Evaluation of Variable Parameter Active Demons Algorithm-Based Diffusion Tensor Imaging on Ventricular Remodeling after Myocardial Infarction and Nursing
}

\author{
Yanhua Liu $\mathbb{D}^{1},{ }^{1}$ Xiaojie Wu $\mathbb{D}^{2},{ }^{2}$ Yu Zhang $\mathbb{D},{ }^{3} \mathrm{Na} \mathrm{Li} \mathbb{D}^{3},{ }^{3}$ and Hua Luan $\mathbb{D}^{3}$ \\ ${ }^{1}$ Respiratory and Critical Care Medicine, The Second Affiliated Hospital of Dalian Medical University, Dalian 116027, China \\ ${ }^{2}$ Operating Room, The Second Affiliated Hospital of Dalian Medical University, Dalian 116027, China \\ ${ }^{3}$ Coronary Care Unit, The Second Affiliated Hospital of Dalian Medical University, Dalian 116027, China
}

Correspondence should be addressed to Hua Luan; 20132412@stu.nun.edu.cn

Received 21 June 2021; Revised 4 August 2021; Accepted 13 August 2021; Published 25 August 2021

Academic Editor: Gustavo Ramirez

Copyright (c) 2021 Yanhua Liu et al. This is an open access article distributed under the Creative Commons Attribution License, which permits unrestricted use, distribution, and reproduction in any medium, provided the original work is properly cited.

\begin{abstract}
This study aimed to explore the effect of diffusion tensor imaging (DTI) image registration algorithm in the evaluation of ventricular remodeling in patients with myocardial infarction and the therapeutic effect of cross-theoretical model rehabilitation exercise care. The dynamic balance coefficient $k$ was introduced to construct the DTI image registration algorithm $k$-AD based on the variable parameter $\mathrm{AD}$ algorithm. Then, it was compared with demons algorithm and $\mathrm{AD}$ algorithm and was applied in 110 patients with acute myocardial infarction. All patients were divided into the 55 cases of experimental group (cross-theoretical model rehabilitation exercise nursing) and 55 cases of control group (routine nursing). The myocardial tissue was labelled as infarction zone (IZ), infarction border zone (IBZ), and noninfarcted zone (NIZ). Finally, the fractional anisotropy (FA) and mean diffusivity (MD) were calculated. The results showed that the mean square error (MSE) of $k$-AD algorithm reduced to 0 after 35 iterations. The descent speed was obviously faster than $\mathrm{AD}$ and demons algorithms. The overlap of eigenvalue-eigenvector pairs (OVL-EEP) value of $k$-AD algorithm $(0.25)$ was smaller than demons $(0.81)$ and AD algorithms $(0.56)(P<0.05)$. The FA value in IZ was notably smaller than IBZ and NIZ, while the MD value was greater than those $(P<0.05)$. After nursing intervention, the FA value of myocardial infarction area in the experimental group was notably higher in contrast to control group, but the MD value was notably lower in contrast to it $(P<0.05)$. In short, the DTI image registration algorithm $k$-AD based on the variable parameter $\mathrm{AD}$ algorithm was remarkably better than the demons and $\mathrm{AD}$ algorithms in convergence and $\mathrm{DTI}$ image registration. FA and $\mathrm{MD}$ values obtained by DTI registration image based on $k$-AD algorithm could clearly indicate the ventricular remodeling after myocardial infarction and the rehabilitation effect of cross-theoretical model rehabilitation exercise nursing intervention.
\end{abstract}

\section{Introduction}

Myocardial infarction refers to ischemic necrosis of the myocardium; the blood flow of the coronary arteries is drastically reduced or interrupted after the occurrence of coronary artery disease. It will cause severe and long-lasting acute ischemia in corresponding myocardium, which eventually leads to avascular necrosis of the myocardium, including acute myocardial infarction and old myocardial infarction $[1,2]$. Ventricular remodeling refers to a series of changes in the size, shape, wall thickness, and tissue structure of the ventricle due to myocardial injury or increased load. It is a process of lesion repair and overall ventricular compensation and secondary pathophysiological reaction. When the body repairs myocardial tissue and ensures heart function, it will activate the body's sympathetic nervous system and renin-angiotensin-aldosterone system. Then, it will produce a large number of neuroendocrine factors to reconstruct the structure of myocardial cells and tissue components [3]. Automatic ventricular remodeling of the body is harmful for human body. It will cause varying degrees of cardiac dysfunction and hemodynamic changes, affect the structural repair and function of the heart, and eventually lead to heart failure and even death. Therefore, 
early diagnosis of the patient's ventricular remodeling is conducive to clinical antiremodeling therapy. The crosstheoretical model is a theoretical model created by American psychology professor James Prochaska in the late 1970s to study individual behavior changes. In clinical nursing, it is patient-centered and adopts motivational interview communication to arouse the intrinsic motivation of patients to change their behavior [4]. Therefore, it was intended to adopt cross-theoretical model rehabilitation exercise nursing to treat patients with myocardial infarction.

DTI is a magnetic resonance imaging technique based on diffusion-weighted imaging [5]. It takes diffusion-weighted gradients in multiple directions to reflect the differences in the diffusion of water molecules in various directions. Then, through the quantitative analysis of the images obtained by diffusion tensor imaging, it reflects the changes in the tissue structure [6]. Current research showed that DTI technology, which was adopted to scan and image the diffusion characteristics of different myocardial regions, could show the ventricular remodeling after myocardial infarction and provide an effective basis for subsequent treatment [7]. The original image may have artifacts, noise, etc., which are not conducive to judgment due to human manipulation and subjective factors. That is why DTI image registration comes into being. However, DTI image registration is much more difficult than traditional scalar registration due to its high dimension and large amount of information of dispersive amplification energy data, so it is necessary to choose a reasonable algorithm for registration. Active demons algorithm is a nonrigid registration method based on the optical flow algorithm. As an improved form of the demons algorithm, it not only further improves the convergence speed of the algorithm but also can register images with larger deformations. However, the algorithm only relies on the average coefficient to adjust the strength of the deformation driving force, which makes the degree of image deformation difficult to control. The inability to manage the accurate registration of large deformation and small deformation regions is likely to cause the disadvantage of low registration accuracy $[8,9]$. Therefore, the AD algorithm was optimized to perform registration analysis on DTI images.

Although AD algorithm was widely used, it still had some limitations. Based on this, the DTI image registration algorithm $k$-AD was constructed based on the variable parameter $\mathrm{AD}$ algorithm by introducing the dynamic balance coefficient $k$. It was compared with the demons algorithm and the AD algorithm and applied in the diagnosis of 110 patients with acute myocardial infarction. The FA value and MA value of image registration in different regions were compared to evaluate the adoption value of DTI image registration based on $\mathrm{AD}$ algorithm in patients with myocardial infarction in terms of ventricular remodeling and postoperative nursing intervention.

\section{Materials and Methods}

2.1. Sample Source. 110 patients with acute myocardial infarction who were admitted to the hospital from October 2018 to February 2020 were selected as the research objects, and they were divided into the experimental group (crosstheoretical model rehabilitation exercise care) and the control group (routine care) based on different postoperative nursing. The research had been approved by the Medical Ethics Committee of Hospital, and the patients had signed the informed consent form.

Inclusion criteria: I, patients with good communication skills; II, patients who had clear consciousness and could cooperate to complete the examination; III, patients with complete visiting records and medical records; IV, patients who were older than eighteen.

Exclusion criteria: I, patients with cognitive dysfunction; II, patients who quitted the test due to personal reasons; III, patients with serious complications such as arrhythmia; IV, patients with congenital heart disease.

2.2. DTI Examination. The $3.0 \mathrm{~T}$ superconducting magnetic resonance imaging instrument produced by Siemens Medical Systems in Germany was adopted for scanning. The patient was scanned from the apex of the heart to the bottom of the heart for three minutes, including the area of myocardial infarction. Scan parameters were as follows: horizons $10 \times 10 \mathrm{~cm}$, layer thickness $3.0 \mathrm{~mm}$, layer spacing $0.5 \mathrm{~mm}$, matrix $251 \times 251$, and number of diffusion directions 26 . Then, the images were transmitted to the workstation, and Functool software was adopted to conduct quantitative analysis of the images before and after the registration by DTI. The tissue layer was classified into IZ, IBZ, and NIZ. FA and $M D$ values were calculated after the region of interest was decided. Then, MSE could be obtained by the contrast of FA between the original image and the registration image.

2.3. Construction of DTI Image Registration Algorithm Based on AD Algorithm. The AD algorithm [10] mainly uses partial information of the original image to transform the image. In the actual deformation iteration, the deformation field will remain continuous in the image. To make the algorithm be continuous in the global scope, Gaussian filter [11] is introduced to smooth the deformation field, and then the deformation vector is as follows:

$$
u=\frac{(M(x, y)-S(x, y)) \nabla S(x, y)}{|\nabla S(x, y)|^{2}+\alpha^{2}(M(x, y)-S(x, y))^{2}}+\frac{(M(x, y)-S(x, y)) \nabla M(x, y)}{|\nabla M(x, y)|^{2}+\alpha^{2}(M(x, y)-S(x, y))^{2}}
$$

In equation $(1),(x, y)$ represents any point of the twodimensional image, $M$ represents the reference image, $S$ represents the floating image, $M(x, y)$ represents the gray value of the reference image within $(x, y)$, and $S(x, y)$ 
represents the gray value of the floating image. $\nabla S(x, y)$ and $\nabla M(x, y)$ represent the gradient of $S$ and $M$, respectively. $\alpha$ represents the averaging coefficient, and $|\nabla S(x, y)|^{2}+\alpha^{2}(M(x, y)-S(x, y))^{2} \geq(2 \alpha \nabla S(x, y))(M(x$, $y)-S(x, y))$. The larger the value $\alpha$, the smaller the deformation vector, the larger the deformation vector, and the better the registration accuracy. However, if the value $\alpha$ is too small, it will be overcorrected, so there is a registration conflict between large deformation zone and small deformation zone. To solve this problem, it introduces the balance coefficient $k$, and then the deformation vector can be expressed as follows:

$$
u=\frac{(M(x, y)-S(x, y)) \nabla S(x, y)}{k^{2}|\nabla S(x, y)|^{2}+\alpha^{2}(M(x, y)-S(x, y))^{2}}+\frac{(M(x, y)-S(x, y)) \nabla M(x, y)}{k^{2}|\nabla M(x, y)|^{2}+\alpha^{2}(M(x, y)-S(x, y))^{2}}
$$

In equation (2), the magnitude of the deformation vector can be affected together by adjusting $\alpha$ and $k$, and $|k \nabla S(x, y)|^{2}+\alpha^{2}(M(x, y)-S(x, y))^{2} \geq$

$(2 \alpha \nabla S(x, y))(M(x, y)-S(x, y))$. When the value $\alpha$ is constant, the smaller the $k$, the larger the deformation vector, and the faster the convergence speed. Then, it introduces the components of the image tensor $W x x, W x y, W x z, W y y$, $W y z$, and $W z z$ as the input channel of the AD algorithm to deform the floating image to the reference image. Then, for each voxel of $(x, y, z)$, the new deformation vector is as follows:

$$
u_{n+1}=G_{\sigma i} *\left(u_{n}+\frac{1}{C}\left(\sum_{c=1}^{C} \frac{\left(\tilde{M}_{\mathrm{cn}}(x, y, z)-S_{c}(x, y)\right) \nabla S_{\mathrm{cn}}(x, y)}{k^{2}\left|\nabla S_{\mathrm{cn}}(x, y, z)\right|^{2}+\alpha^{2}\left|\tilde{M}_{\mathrm{cn}}(x, y, z)-S_{c}(x, y, z)\right|^{2}}+\frac{\left(\tilde{M}_{\mathrm{cn}}(x, y, z)-S_{c}(x, y, z)\right) \nabla \tilde{M}_{\mathrm{cn}}(x, y, z)}{k^{2}|\nabla M(x, y, z)|^{2}+\alpha^{2}\left|\tilde{M}_{\mathrm{cn}}(x, y, z)-S_{c}(x, y, z)\right|^{2}}\right)\right) .
$$

In equation (3), * represents the convolution operation, $C$ represents the number of input channels, $\widetilde{M}_{\mathrm{cn}}$ represents the reset channel $c, G_{\sigma i}$ represents the Gaussian filter with variance, $N$ represents the maximum number of iterations, and $i$ represents an integer taken upward. When $\sigma$ gradually becomes smaller, the registration begins to strongly restrict deformation and correct displacement. Then, it manually selects a value $\alpha^{2}$ within a suitable range and adjusts the dynamic balance coefficient $k_{i}^{2}$ for reasonable registration when the algorithm converges, which can be expressed as follows:

$$
u_{n+1}=G_{\sigma i} *\left(u_{n}+\frac{1}{C}\left(\sum_{c=1}^{C} \frac{\left(\tilde{M}_{\mathrm{cn}}(x, y, z)-S_{c}(x, y)\right) \nabla S_{\mathrm{cn}}(x, y)}{k_{i}^{2}\left|\nabla S_{\mathrm{cn}}(x, y, z)\right|^{2}+\alpha^{2}\left|\tilde{M}_{\mathrm{cn}}(x, y, z)-S_{c}(x, y, z)\right|^{2}} \sigma^{2}+\frac{\left(\tilde{M}_{\mathrm{cn}}(x, y, z)-S_{c}(x, y, z)\right) \nabla \tilde{M}_{\mathrm{cn}}(x, y, z)}{k_{i}^{2}|\nabla M(x, y, z)|^{2}+\alpha^{2}\left|\tilde{M}_{\mathrm{cn}}(x, y, z)-S_{c}(x, y, z)\right|^{2}}\right)\right)
$$

In equation (4), $k_{i}{ }^{2}$ represents the dynamic balance coefficient, $i=\lceil 4 n / N\rceil$, and \lceil\rceil means it takes the upward value. The proposed algorithm can use a smaller dynamic balance coefficient in the initial stage of registration, to give the registration faster convergence performance and gradually increase the dynamic balance coefficient to reduce the registration error. To achieve the effect of improving speed and accuracy at the same time, it is set as $k$-AD. The specific process is shown in Figure 1. First, the tensor component is input to the reference image and the floating image, and the parameters $\alpha, \sigma$, and $k$ are initialized. $\sigma$ should be adjusted first, if it calculates the deformation vector of the floating image after updating $k$, Gaussian smoothing is conducted for the displacement field, trilinear interpolation is conducted for the moving image, then tensor redirection is conducted for each voxel, and finally the image after registration is output.
2.4. DTI Image Registration Quality Evaluation Index. Demons algorithm and $\mathrm{AD}$ algorithm are adopted to be compared with the algorithm $k-\mathrm{AD}$, and the OVL-EEP [12] is taken to evaluate the image quality of the algorithm registration. The equation is as follows:

$$
\mathrm{OVL}=\frac{1}{N} \sum_{i=1}^{N} \frac{\sum_{j=1}^{3} \lambda_{j}^{i} \lambda_{j}^{i^{\prime}}\left(e_{j}^{i} e_{j}^{i^{\prime}}\right)^{2}}{\sum_{j=1}^{3} \lambda_{j}^{i} \lambda_{j}^{i^{\prime}}}
$$

In equation (5), $\lambda_{j}^{i}, \lambda_{j}^{i^{\prime}}, e_{j}^{i}$, and $e_{j}^{i^{\prime}}$ represent the $j$-th feature vector pair of the $i$-th voxel of the dispersion tensor. The larger the value of OVL-EEP, the better the image registration effect of the algorithm.

2.5. Statistical Method. The data processing was analyzed by SPSS19.0 version statistical software, the measurement data were expressed as mean plus or minus standard deviation 


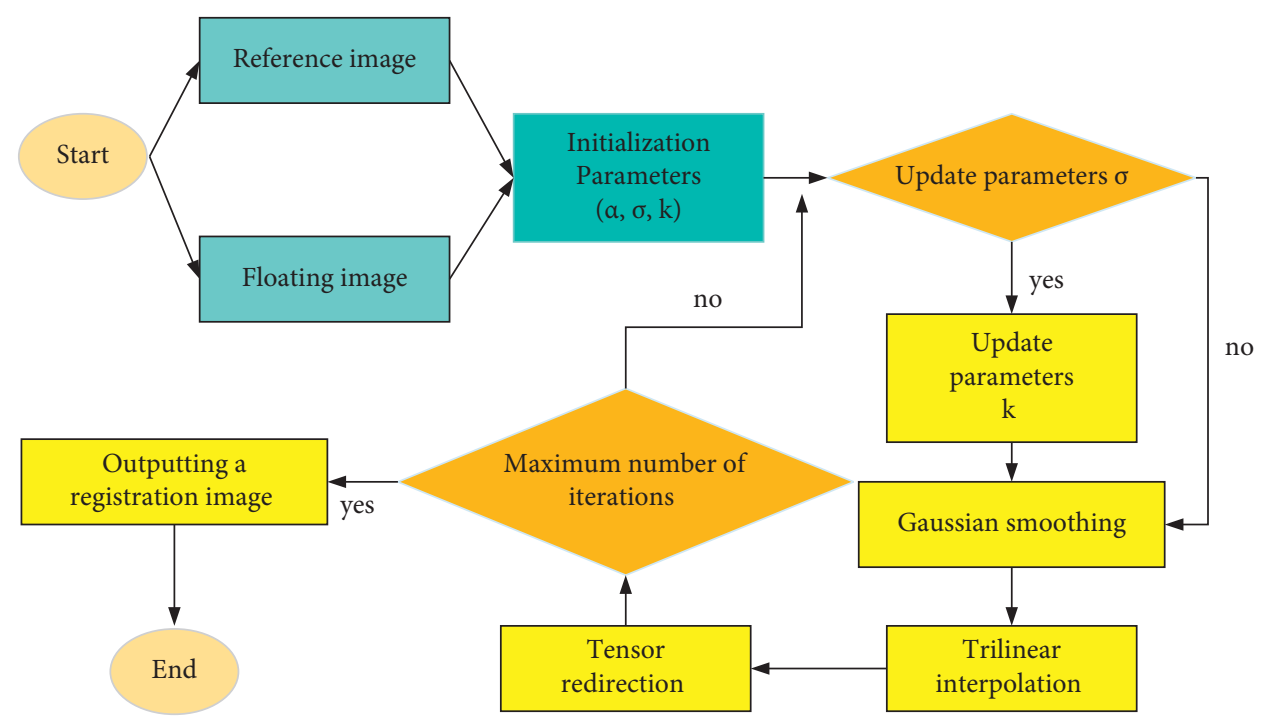

FIGURE 1: DTI image registration algorithm based on variable parameter $\mathrm{AD}$ algorithm.

$(\bar{x} \pm s)$, and the count data were expressed as percentage (\%). The OVL-EEP values of demons, $\mathrm{AD}$, and $k$-AD algorithms were compared via $t$-test. The pairwise comparison between FA values and MD values of IZ, IBZ, and NIZ were conducted by variance analysis. The difference was statistically significant when $P<0.05$.

\section{Results}

3.1. Contrast of MSE Convergence of the Algorithms. The number of iterations was set as $0,5,10,15,20,25,30,35$, and 40. In Figure 2, the MSE of the three algorithms all decreased with the increase of the number of iterations, while the MSE of $k$-AD algorithm was reduced to 0 at the 35 th iterations, and the descending speed was obviously faster than that of $\mathrm{AD}$ algorithm and demons algorithm.

3.2. Contrast of DTI Image Registration Effect of Algorithms. Figure 3 shows that the OVL-EEP of the demons, AD, and $k$ $\mathrm{AD}$ algorithm was $0.81,0.56$, and 0.25 , respectively. The OVL-EEP value of $k$-AD algorithm was notably lower in contrast to demons algorithm and AD algorithm, $P<0.05$. The OVL-EEP value of AD algorithm was notably lower in contrast to demons algorithm, $P<0.05$. Figure 4 shows the DTI image registration effect of different algorithms on FA and MD value pseudocolor image. It was obvious that $k$-AD algorithm registration image clarity was higher, organization detail display was more perfect, and the image registration quality was the best with the highest accuracy.

\subsection{Contrast of FA Values in Infarction Zone, Infarction} Border Zone, and Noninfarcted Zone of Patients. Figure 5 shows that the FA value of the IZ, IBZ, and NIZ was $0.117 \pm 0.038,0.226 \pm 0.071$, and $0.374 \pm 0.054$. Among them, the FA value of IZ was notably lower in contrast to IBZ and NIZ, with statistically obvious difference $(P<0.05)$. The FA value in IZ was notably smaller in contrast to NIZ area, and the difference was statistically notable $(P<0.05)$. Figure 6 shows pseudocolor map of FA values in different areas. The myocardial fiber bundles in Figure 6(a) were relatively rich and neatly arranged. The shape was continuous and tight, and the anisotropy at different levels spread significantly. The myocardial fiber bundles in Figure 6(b) were relatively sparse and arranged relatively loosely. The shape was not neat, and the anisotropic diffusion at different levels was obviously weakened. The myocardial fiber bundles in Figure 6(c) were greatly reduced, the shape was chaotic, and the arrangement was not integrated. There were no fiber bundles in some areas, and the anisotropic diffusion was weakened.

3.4. Contrast of MD Values in Infarction Zone, Infarction Border Zone, and Noninfarcted Zone of Patients. In Figure 7, the $M D$ value in $I Z$ was $(1.318 \pm 0.172) \times 10^{-3} \mathrm{~mm}^{2} / \mathrm{s}$, that in IBZ was $(1.026 \pm 0.171) \times 10^{-3} \mathrm{~mm}^{2} / \mathrm{s}$, and that in NIZ was $(0.765 \pm 0.154) \times 10^{-3} \mathrm{~mm}^{2} / \mathrm{s}$. Among them, the MD value in IZ was notably greater in contrast to IBZ and NIZ, with statistically notable difference $(P<0.05)$. The MD value in IZ was obviously greater in contrast to NIZ, and the difference was also notable $(P<0.05)$. Figure 8 shows a pseudocolor map of $\mathrm{MD}$ values in different regions. The myocardial fiber bundles in Figure 8(a) were abundant, the overall arrangement was orderly, and the different layers of anisotropy spread obviously. The area of myocardial fiber bundles in Figure 8(b) was reduced, and the degree of anisotropic diffusion at different layers was notably weakened. The overall shape of the myocardial fiber bundles in Figure 8(c) was chaotic, and there were no fiber bundles in some areas, and the anisotropic diffusion was weakened.

3.5. Comparison of FA and MD Values of Patients' Infarction Zone, Infarction Border Zone, and Noninfarcted Zone after Nursing Intervention. Figure 9 reveals that the FA value in IZ of myocardial infarction in the experimental group after 


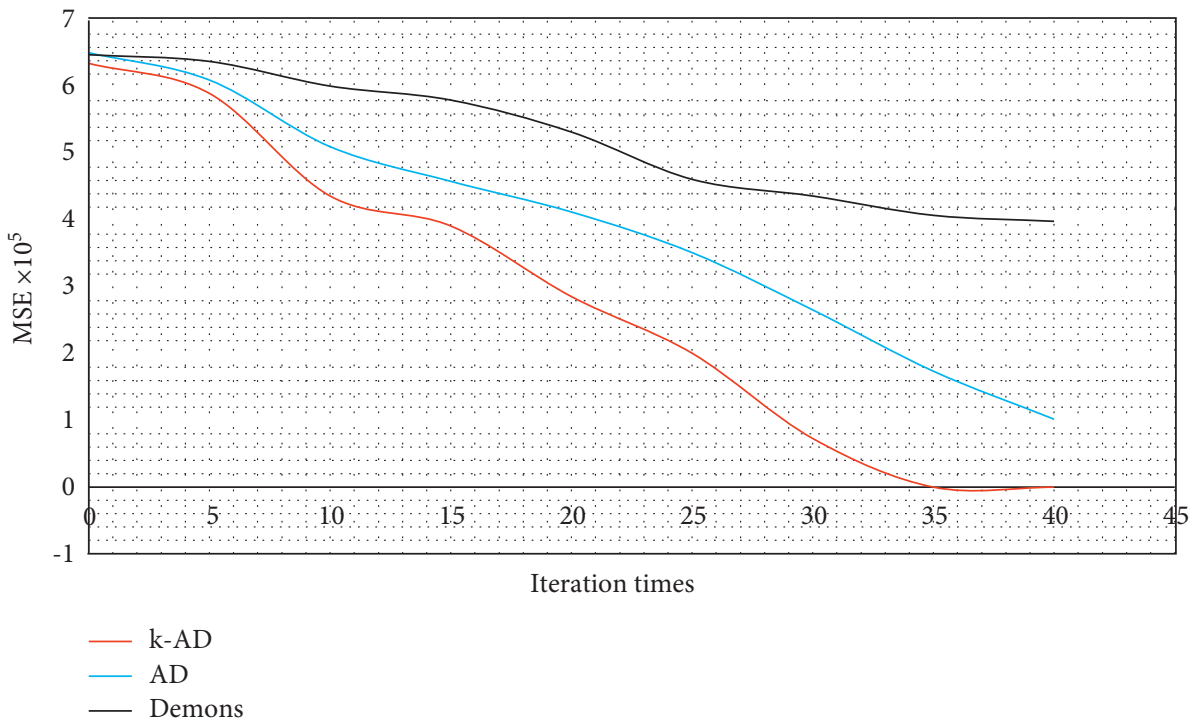

FIgURE 2: Contrast of MSE under different iteration times of three algorithms.

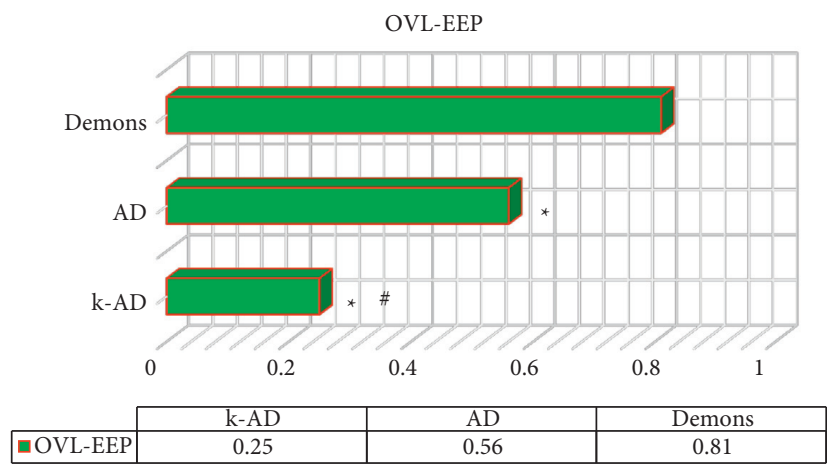

Figure 3: The OVL-EEP value of three algorithms for registration of DTI images of patients with myocardial infarction. Note. * and \# indicate that the difference was statistically notable in contrast to demons algorithm and AD algorithm, respectively $(P<0.05)$.
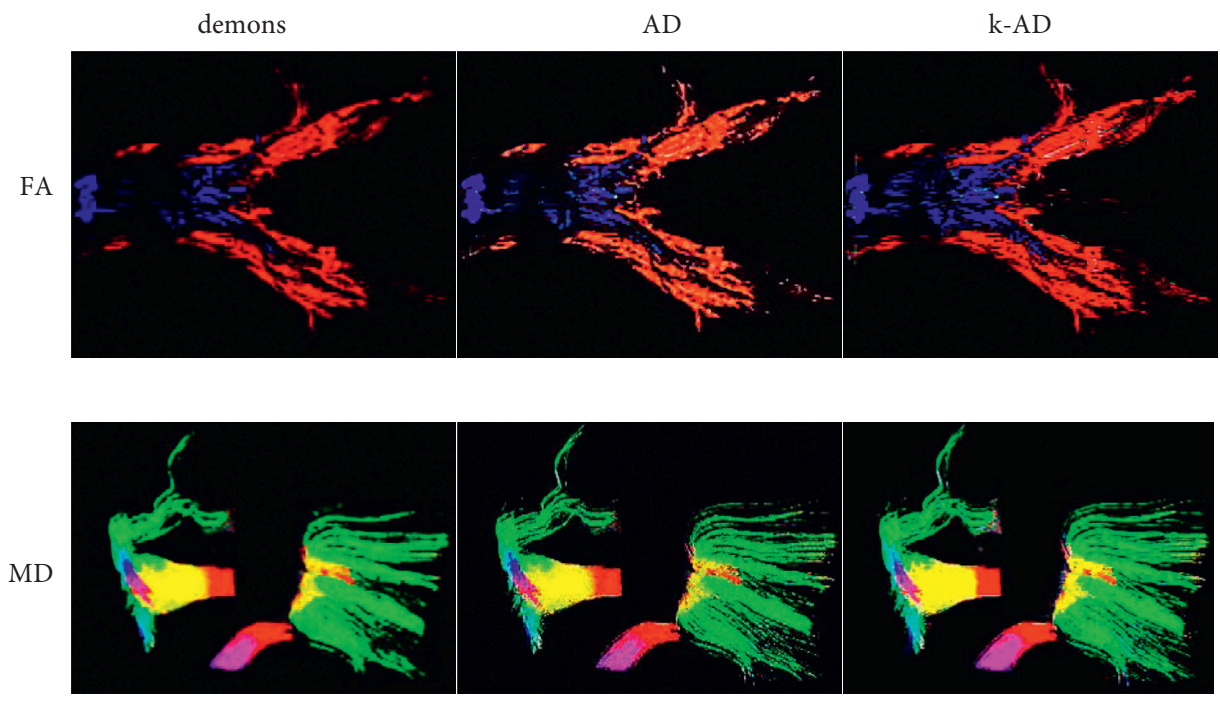

FIgURE 4: The FA and MD value pseudocolor image registration effect of three algorithms for patient DTI image registration. 


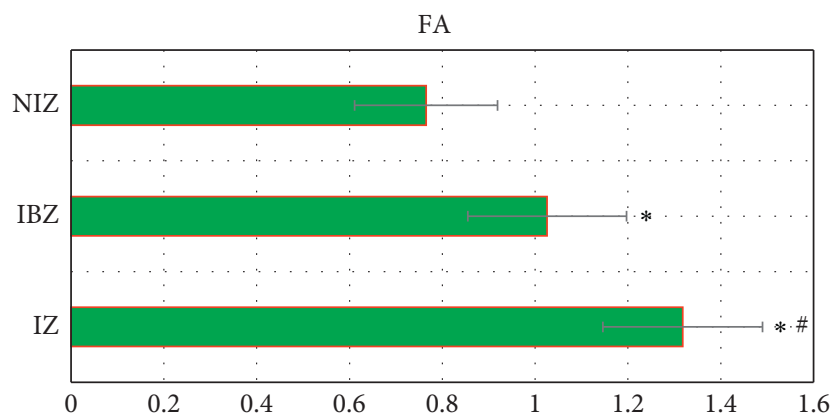

Figure 5: Contrast of FA values in different areas of patients with myocardial infarction. Note. * and \# indicate that the difference was statistically notable in contrast to NIZ and IBZ, respectively $(P<0.05)$.

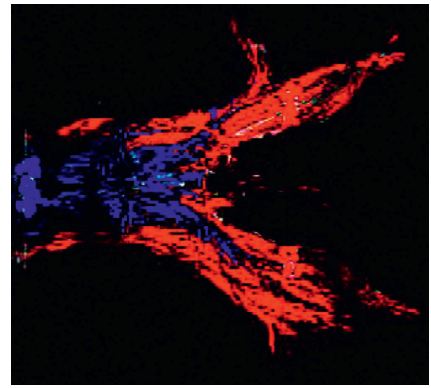

(a)

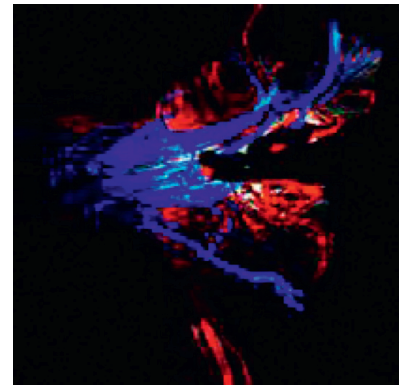

(b)

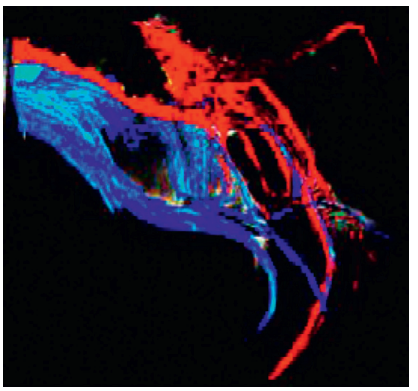

(c)

Figure 6: Pseudocolor map of FA values in different regions. (a) A pseudocolor map of FA value in the NIZ; (b) a pseudocolor map of FA value in the IBZ; (c) a pseudocolor map of FA value in the IZ.

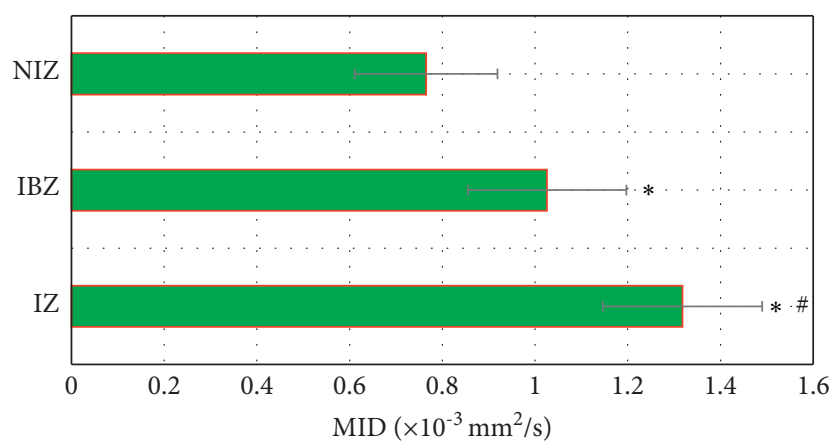

FIGURE 7: Contrast of MD in different areas of myocardial infarction. Note. * and \# indicate that the difference was statistically notable in contrast to NIZ and IBZ, respectively $(P<0.05)$.

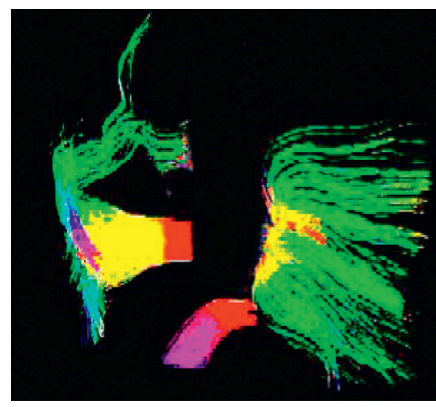

(a)

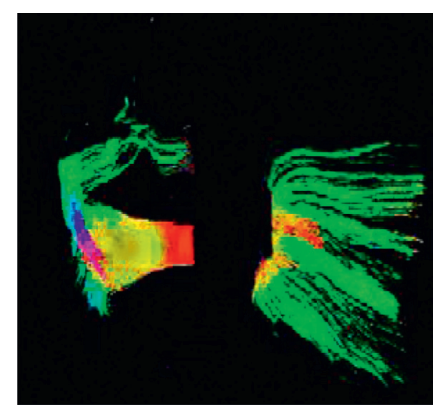

(b)

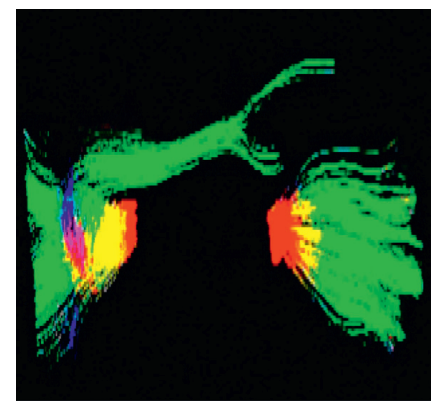

(c)

Figure 8: Pseudocolor map of MD values in different regions. (a) A pseudocolor map of MD value in NIZ; (b) pseudocolor map of MD value in IBZ; (c) a pseudocolor map of MD value in IZ. 

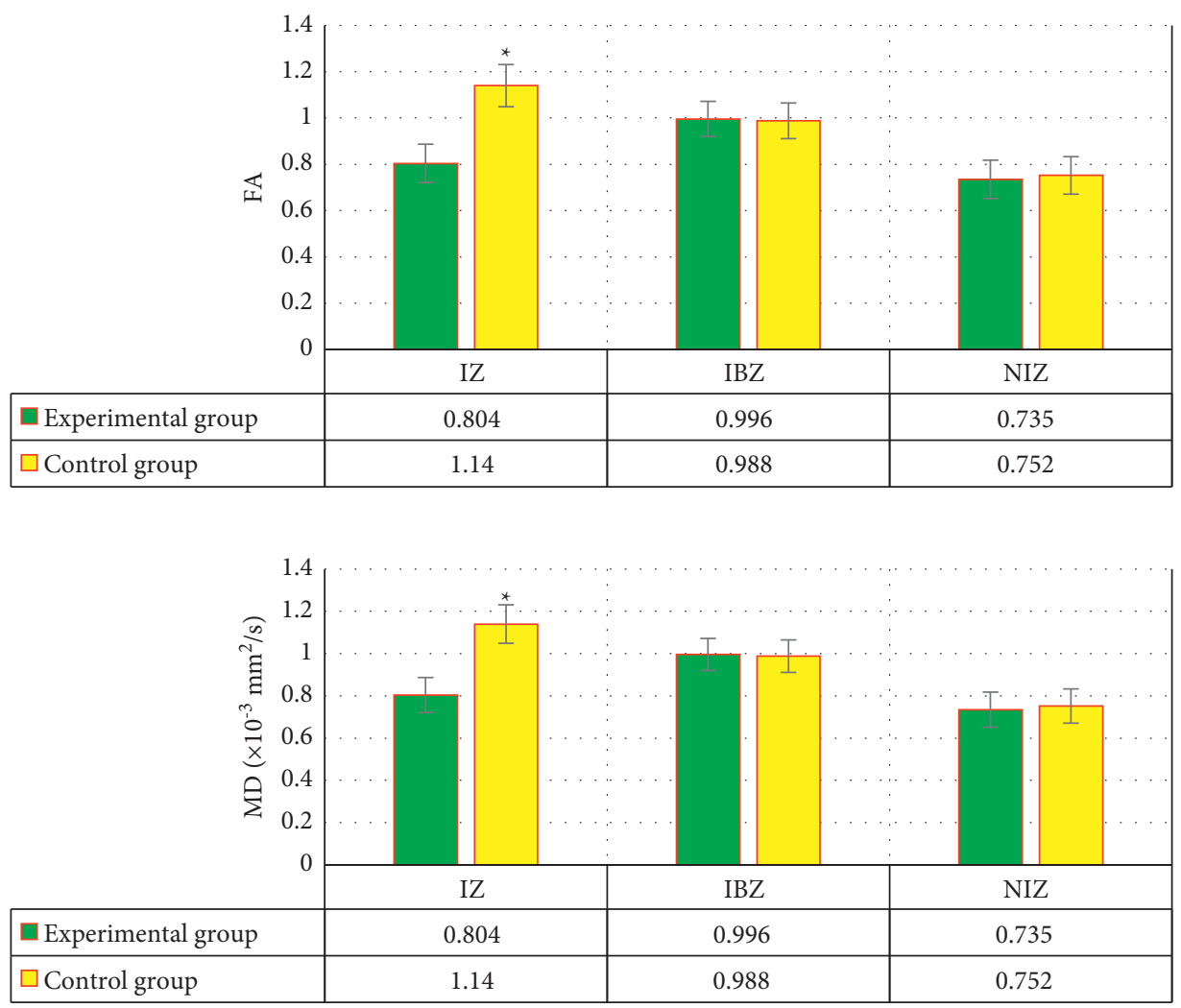

FIGURE 9: Contrast of FA and MD values in different areas of patients with myocardial infarction after nursing intervention. (a) The FA value in different areas; (b) the MD value in different areas. Note. $*$ and \# indicate that the difference was statistically notable in contrast to NIZ and IBZ, respectively $(P<0.05)$.

nursing intervention was remarkably higher in contrast to control group $(P<0.05)$. The MD value in IZ of the experimental group after nursing intervention was notably lower than the control group $(P<0.05)$.

\section{Discussion}

Due to the complexity and high order of tensor data, DTI image registration is much more difficult than traditional medical image registration. Therefore, it is crucial to select an appropriate image registration algorithm [13]. The DTI image registration algorithm $(k-\mathrm{AD})$ based on the variable parameter $\mathrm{AD}$ algorithm was constructed by introducing the dynamic balance coefficient $k$. Then, it was compared with the demons algorithm and the $\mathrm{AD}$ algorithm. The results showed that the MSE of $k$-AD algorithm was reduced to 0 after 35 iterations, and the descending speed was obviously faster than that of $\mathrm{AD}$ and demons algorithms. It meant that the $k$-AD algorithm had a better convergence performance and could help to obtain higher registration accuracy under the same number of iterations [14]. The OVL-EEP value of $k$ $\mathrm{AD}$ algorithm was obviously less than that of demons and $\mathrm{AD}$ algorithms, and the OVL-EEP value of AD algorithm was less than demons algorithm $(P<0.05)$, which was similar to the findings of Federico et al. [15]. It suggested that $k$-AD algorithm was better than demons algorithm and $\mathrm{AD}$ algorithm for DTI image registration. From the perspective of image registration, the $k$-AD algorithm had a higher image definition and the best image quality, which was corresponding to the quantitative data results.

The FA value in IZ was notably lower than in IBZ and NIZ, and the FA value in IZ was notably lower than in NIZ $(P<0.05)$, which was different from the study results of Okura et al. (2019) [16]. The results showed that the FA values obtained based on DTI scanning image registration were obviously different from those in IZ, NIZ, and IBZ, and the FA values in IZ were much smaller than that in NIZ. It could clearly indicate the status of ventricular remodeling after myocardial infarction. The MD value in $\mathrm{IZ}$ was greater than those in IBZ and NIZ, and the MD value in IZ was notably greater than that in NIZ $(P<0.05)$. It also indicated that the MD value obtained based on DTI scan image registration could evaluate the ventricular remodeling of patients after myocardial infarction. After nursing intervention, $\mathrm{FA}$ value and $\mathrm{MD}$ value in $\mathrm{IZ}$ of the experimental group were remarkably higher in contrast to control group $(P<0.05)$, which was different from the study results of Wang et al. (2016) [17]. The reason may be that the selected number and scope of patient samples were different, which leaded to different results. Thus, it could be concluded that compared with conventional nursing, cross-theoretical model rehabilitation exercise nursing intervention had a better promoting effect on postoperative rehabilitation of patients. 


\section{Conclusion}

Based on AD algorithm, the dynamic balance coefficient $k$ was introduced to construct the DTI image registration algorithm $k$-AD based on the variable parameter $\mathrm{AD}$ algorithm. Then, it was compared with demons algorithm and $\mathrm{AD}$ algorithm. The DTI image registration algorithm $k$-AD was applied in the preoperative and postoperative nursing intervention evaluation of patients with myocardial infarction. The results showed that the $k$-AD algorithm based on the variable parameter active demons algorithm had an excellent effect on DTI image registration. Moreover, DTI registration image parameters FA and $\mathrm{MD}$ values could clearly indicate the ventricular remodeling effect after myocardial infarction, as well as the effect of rehabilitation exercise nursing intervention across the theoretical model. However, the sample size selected in this study is small and the source is single, which may make the results have certain limitations. Moreover, the initial parameters of the algorithm need to be set manually and need to be adjusted for different sample sizes. Later, it will be considered to increase the sample size and discuss the specific practicality of the AD algorithm. In conclusion, the results of this study provided theoretical help for clinical DTI image registration.

\section{Data Availability}

No data were used to support this study.

\section{Conflicts of Interest}

The authors declare no conflicts of interest.

\section{Authors' Contributions}

Yanhua Liu and Xiaojie $\mathrm{Wu}$ contributed equally to this work.

\section{References}

[1] M. Boudier-Revéret, M. Y. Hsiao, S. G. Shyu, and C. Min Cheol, "Injury of corticospinal tract and corticoreticular pathway caused by high-voltage electrical shock: a case report," BMC Neurology, vol. 20, pp. 1-4, 2020.

[2] P. Deedwania and G. W. Huang, "An update on antithrombotic therapy in atrial fibrillation: the role of newer and emergent drugs," Reviews in Cardiovascular Medicine, vol. 13, no. 2-3, pp. 89-104, 2019.

[3] G. Koracevic, M. Stojkovic, D. Lovic et al., "Should cushing's syndrome be considered as a disease with high cardiovascular risk in relevant guidelines?" Current Vascular Pharmacology, vol. 18, no. 1, pp. 12-24, 2020.

[4] S. Hosseini, A. Fazlinezhad, M. Jalalyazdi, and A. Gharaee, "Diastolic function changes during stress echocardiography in hypertensive patients," Razavi International Journal of Medicine, vol. 5, no. 2, Article ID e42876, 2017.

[5] G. M. Annich, O. Zaulan, M. Neufeld, D. Wagner, and M. M. Reynolds, "Thromboprophylaxis in extracorporeal circuits: current pharmacological strategies and future directions," American Journal of Cardiovascular Drugs, vol. 17, no. 6, pp. 425-439, 2017.
[6] F. S. Taccone, I. Baar, C. De Deyne et al., "Neuroprognostication after adult cardiac arrest treated with targeted temperature management: task force for Belgian recommendations," Acta Neurologica Belgica, vol. 117, no. 1, pp. 3-15, 2017.

[7] L. Lenchik, L. Heacock, A. A. Weaver et al., "Automated segmentation of tissues using CT and MRI: a systematic review," Academic Radiology, vol. 26, no. 12, pp. 1695-1706, 2019.

[8] E. Widerström-Noga, "Neuropathic pain and spinal cord injury: phenotypes and pharmacological management," Drugs, vol. 77, no. 9, pp. 967-984, 2017.

[9] J. Flores and F. Sasangohar, "Towards a theoretical model of aggregate fatigue in nursing," Proceedings of the Human Factors and Ergonomics Society - Annual Meeting, vol. 62, no. 1, pp. 1679-1683, 2018.

[10] D. G. León, M. López-Yunta, J. M. Alfonso-Almazán et al., "Three-dimensional cardiac fibre disorganization as a novel parameter for ventricular arrhythmia stratification after myocardial infarction," EP Europace, vol. 21, no. 5, pp. 822-832, 2019.

[11] H. S. Roshdy, I. I. El-Dosouky II, and M. H. Soliman, "Highrisk inferior myocardial infarction: can speckle tracking predict proximal right coronary lesions?" Clinical Cardiology, vol. 41, no. 1, pp. 104-110, 2018.

[12] Y. Tian, Z. Liu, Z. Tang et al., "Radiomics analysis of DTI data to assess vision outcome after intravenous methylprednisolone therapy in Neuromyelitis optic neuritis," Journal of Magnetic Resonance Imaging, vol. 49, no. 5, pp. 1365-1373, 2019.

[13] A. Tornyos, D. Kehl, F. D’Ascenzo, and A. Komócsi, "Risk of myocardial infarction in patients with long-term nonvitamin $\mathrm{K}$ antagonist oral anticoagulant treatment," Progress in Cardiovascular Diseases, vol. 58, no. 5, pp. 483-494, 2016.

[14] Y. Lao, B. Nguyen, S. Tsao et al., "A T1 and DTI fused 3D corpus callosum analysis in MCI subjects with high and low cardiovascular risk profile," NeuroImage: Clinica, vol. 14, pp. 298-307, 2017.

[15] B. Federico, A. Francesco, M. Silvia et al., "Application of diffusion tensor imaging (DTI) and MR-tractography in the evaluation of peripheral nerve tumours: state of the art and review of the literature," Acta BioMedica: Atenei Parmensis, vol. 90, no. 5, p. 68, 2019.

[16] H. Okura, Y. Saito, Y. Saito et al., "Frequency and prognostic impact of intravascular imaging-guided urgent percutaneous coronary intervention in patients with acute myocardial infarction: results from J-MINUET," Heart and Vessels, vol. 34, no. 4, pp. 564-571, 2019.

[17] Y. Wang, W. Cai, L. Wang et al., "Evaluation of the differences of myocardial fibers between acute and chronic myocardial infarction: application of diffusion tensor magnetic resonance imaging in a rhesus monkey model," Korean Journal of Radiology, vol. 17, no. 5, pp. 725-733, 2016. 